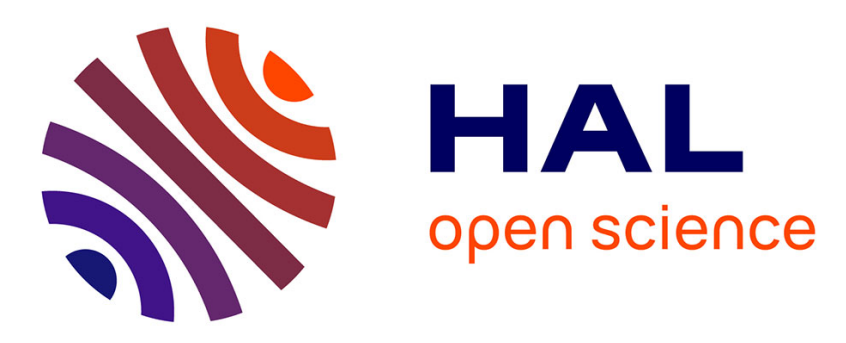

\title{
Ultrashort high energy electron bunches from tunable surface plasma waves driven with laser wavefront rotation
}

S Marini, P S Kleij, F Pisani, F Amiranoff, M Grech, A Macchi, Michèle Raynaud, C Riconda

\section{To cite this version:}

S Marini, P S Kleij, F Pisani, F Amiranoff, M Grech, et al.. Ultrashort high energy electron bunches from tunable surface plasma waves driven with laser wavefront rotation. Physical Review E , 2021, 103, 10.1103/physreve.103.1021201 . hal-03515445

\section{HAL Id: hal-03515445 \\ https://hal.science/hal-03515445}

Submitted on 6 Jan 2022

HAL is a multi-disciplinary open access archive for the deposit and dissemination of scientific research documents, whether they are published or not. The documents may come from teaching and research institutions in France or abroad, or from public or private research centers.
L'archive ouverte pluridisciplinaire HAL, est destinée au dépôt et à la diffusion de documents scientifiques de niveau recherche, publiés ou non, émanant des établissements d'enseignement et de recherche français ou étrangers, des laboratoires publics ou privés. 


\title{
Ultrashort high energy electron bunches from tunable surface plasma waves driven with laser wavefront rotation
}

\author{
S. Marini $\odot,{ }^{1,2}$ P. S. Kleij $\odot,{ }^{1,2,3}$ F. Pisani $\odot,{ }^{3}$ F. Amiranoff, ${ }^{2}$ M. Grech $\odot,{ }^{2}$ A. Macchi $\odot,{ }^{4,3}$ M. Raynaud $\odot,{ }^{1}$ and C. Riconda $\odot^{2, *}$ \\ ${ }^{1}$ LSI, CEA/DRF/IRAMIS, CNRS, École Polytechnique, Institut Polytechnique de Paris, F-91128 Palaiseau, France \\ ${ }^{2}$ LULI, Sorbonne Université, CNRS, CEA, École Polytechnique, Institut Polytechnique de Paris, F-75252 Paris, France \\ ${ }^{3}$ Enrico Fermi Department of Physics, University of Pisa, largo Bruno Pontecorvo 3, 56127 Pisa, Italy \\ ${ }^{4}$ National Institute of Optics, National Research Council (CNR/INO), Adriano Gozzini laboratory, 56124 Pisa, Italy
}

(Received 31 July 2020; revised 22 September 2020; accepted 15 January 2021; published 15 February 2021)

\begin{abstract}
We propose to use ultrahigh intensity laser pulses with wave-front rotation (WFR) to produce short, ultraintense surface plasma waves (SPW) on grating targets for electron acceleration. Combining a smart grating design with optimal WFR conditions identified through simple analytical modeling and particle-in-cell simulation allows us to decrease the SPW duration (down to a few optical cycles) and increase its peak amplitude. In the relativistic regime, for $I \lambda_{0}^{2}=3.4 \times 10^{19} \mathrm{~W} / \mathrm{cm}^{2} \mu \mathrm{m}^{2}$, such SPW are found to accelerate high charge (few $10 \mathrm{~s}$ of $\mathrm{pC}$ ), high energy (up to $70 \mathrm{MeV}$ ), and ultrashort (few fs) electron bunches.
\end{abstract}

DOI: 10.1103/PhysRevE.103.L021201

Surface plasmon polaritons, also known as surface plasma waves (SPW) in free electron media, are highly localized electromagnetic field structures with the ability to confine and enhance light in subwalength regions at the interface between two media [1-4]. Their unique properties have made them ideal candidates for applications in a broad range of research fields, from biochemical sensing $[5,6]$ to the design of small photonic devices [7,8].

The excitation of SPW by micrometric wavelength $\left(\lambda_{0}=\right.$ $0.8 \mu \mathrm{m}$ ) femtosecond (fs) laser pulses irradiating solid targets has been demonstrated as a strategy to enhance secondary emission of radiation and particles. In the low intensity regime, from few $\mathrm{GW} / \mathrm{cm}^{2}$ to tens of $\mathrm{TW} / \mathrm{cm}^{2}$, surface plasmon polaritons have led to harmonic emission [9-11] and the production of photoelectron bunches at energies up to few 100s eV [12,13]. The advent of table-top, 10s TW, fs lasers allowed on-target irradiance $I_{0} \lambda_{0}^{2} \gtrsim 10^{18} \mathrm{~W} / \mathrm{cm}^{2} \mu \mathrm{m}^{2}$. In this ultrahigh intensity (UHI) regime, any target material quickly turns into a plasma, and electrons reach relativistic quiver velocities in the intense laser field. SPW then become of interest not only as unexplored nonlinear plasma modes but also for their capability of accelerating electrons, being waves with a longitudinal electric field component and slightly subluminal phase speed. Simulations and experiments have indeed shown that relativistic SPW can accelerate high charge, ultrashort electron bunches along the target surface [14-25], with energies largely exceeding their quiver energy and spatiotemporal correlation with extreme ultraviolet (XUV) harmonic emission [26].

In a recent paper, Pisani et al. [27] showed through electromagnetic simulations in the linear optics (low intensity) regime that using wave-front rotation (WFR) on the driving laser pulse could help generate more intense, shorter SPW.

*caterina.riconda@ sorbonne-universite.fr
WFR is a technique used on fs lasers to induce a rotation of the successive laser wave fronts, thus leading to a time-varying incidence angle of the laser impinging onto a target. Since SPW on a grating are excited for a well-defined value of this angle, using WFR allows for the SPW excitation only over a very short time, leading to the generation of near singlecycle SPW; an enhancement of the excited SPW was also found.

In this Letter, we demonstrate how these effects can be harnessed in the UHI regime, and WFR can be used to drive tunable, ultrashort, ultraintense SPW able to generate near single-cycle, highly energetic electron bunches. The optimal WFR conditions are identified using both analytical modeling and kinetic (particle-in-cell, PIC) simulations. They allow for a significant increase of both the SPW amplitude and the electron energy by up to $65 \%$ with respect to the case without WFR. A careful design of the grating target allows for an additional increase (by 25\%) of the electron maximum energy. Electron bunches with several $10 \mathrm{~s}$ of $\mathrm{MeV}$ energy and $10 \mathrm{~s}$ of $\mathrm{pC}$ charge are predicted considering currently available table-top laser parameters.

The interaction setup considered throughout this work is depicted in Fig. 1. A UHI laser pulse impinges onto an overdense plasma with density $n \gg n_{c}$, with $n_{c}=\epsilon_{0} m_{e} \omega_{0}^{2} / e^{2}$ being the critical density at the laser frequency $\omega_{0}=2 \pi c / \lambda_{0}$, $c$ the vacuum speed of light, $\epsilon_{0}$ the vacuum permittivity, and $m_{e}$ and $-e$ the electron mass and charge. To resonantly excite a SPW at the vacuum-plasma interface, the target surface is partially modulated, and the laser incidence angle $\left(\theta_{0}\right)$ is chosen such that $\sin \theta_{0}=\sqrt{\left(n / n_{c}-1\right) /\left(n / n_{c}-2\right)}-\lambda_{0} / d$, with $d$ being the target periodicity [1]. The resulting SPW is excited at the laser frequency $\omega=\omega_{0}$ and satisfies the dispersion relation (nonrelativistic cold-fluid model [28]) $c^{2} k_{\mathrm{sPw}}{ }^{2} / \omega^{2}=$ $\left(\omega_{p}^{2} / \omega^{2}-1\right) /\left(\omega_{p}^{2} / \omega^{2}-2\right)$, with $k_{\mathrm{SPW}}$ being the SPW wave number and $\omega_{p}=\sqrt{e^{2} n /\left(\epsilon_{0} m_{e}\right)}$ the electron plasma frequency. For $n \gg n_{c}$, the SPW phase and group velocities are 


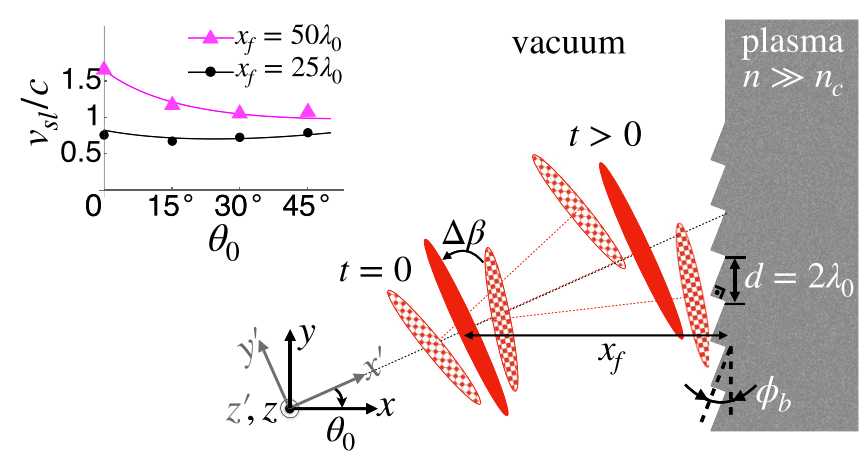

FIG. 1. Interaction setup: the central laser wave fronts are shown at best focus $(t=0)$ and striking the target $(t>0)$. Due to WFR, setting the target at a distance $x_{f}$ from best focus leads to a "sliding focus" effect, with the maximum on-target intensity sliding in the $y$ direction at a velocity $v_{\mathrm{sl}}$. The upper left insert compares $v_{\mathrm{sl}}$ from Eq. (3) (solid lines) with measures from PIC simulations ( symbols) for $x_{f}=25 \lambda_{0}$ (black circles) and $x_{f}=50 \lambda_{0}$ ( magenta triangles).

slightly subluminal: $v_{\phi} \rightarrow c\left[1-n_{c} /(2 n)\right]$ and $v_{g} \rightarrow c[1-$ $\left.3 n_{c} /(2 n)\right]$.

As shown in Fig. 1, the target is located at a distance $x_{f}$ from the laser best focus, which together with WFR allows for a "sliding focus" effect, i.e., a displacement in time of the pulse intensity peak along the target surface. If the sliding focus velocity $v_{\mathrm{sl}}$ is close to the SPW velocity, the latter will be driven more efficiently. To estimate $v_{\mathrm{sl}}$, let us recall that at focus, the electric field of a pulse with WFR can be written as [29]

$$
E\left(y^{\prime}, t\right)=E_{0} f(t) F\left(y^{\prime}\right) \exp \left[i \phi\left(y^{\prime}, t\right)\right] .
$$

Here $E_{0}$ is the maximum electric field, $f(t)$ and $F\left(y^{\prime}\right)$ are the electric field temporal and transverse (in our two-dimensional [2D] configuration) spatial envelope, and the spatiotemporal phase is

$$
\phi\left(y^{\prime}, t\right)=\omega_{0} t\left(1-\Omega_{\beta} y^{\prime} / c\right) .
$$

The linear dependence in $y^{\prime} t$ leads to an instantaneous angle of propagation of light $\beta(t) \simeq-\left(c / \omega_{0}\right) \partial \phi / \partial y^{\prime}=\Omega_{\beta} t$ increasing linearly with time, with $\Omega_{\beta}$ being the WFR velocity. In Fig. $1, \Omega_{\beta}>0$ is considered, only the central wave fronts are represented, and angles are exaggerated for illustration purposes. The main angle of incidence $\theta_{0}$, defined as that of the central wavefront, is chosen as the resonant angle for exciting the SPW. Successive wave fronts are then shifted by an angle $\Delta \beta=\Omega_{\beta} \lambda_{0} / c$ henceforth referred to as the WFR parameter. As a result, each successive wave front will strike the target at a slightly different location along the $y$ direction, leading to the apparent sliding velocity of the pulse on the target. For ultrashort pulses and/or the central wave fronts, we obtain a constant sliding velocity:

$$
v_{\mathrm{sl}} \simeq \frac{\Delta \beta x_{f} / \lambda_{0}}{\cos ^{2} \theta_{0}+\sin \theta_{0} \Delta \beta x_{f} / \lambda_{0}} c .
$$

As shown in the insert of Fig. 1 (for $\Delta \beta=33 \mathrm{mrad}$ ), Eq. (3) is found to be in good agreement with measurements from PIC simulations. ${ }^{1}$

The sign and value of the WFR parameter $\Delta \beta$ affects the duration and amplitude of the excited SPW [27]. Indeed, when the sliding velocity is along the direction of propagation of the SPW, the excited wave can increase its amplitude while maintaining a short duration. Additional tunability can be obtained by calculating an optimal value of the WFR parameter $\Delta \beta_{\text {opt }}$ such that the sliding velocity $v_{\mathrm{sl}}$ coincides with the SPW velocity $\simeq c$; this leads to

$$
\Delta \beta_{\mathrm{opt}} \simeq \frac{\lambda_{0}}{x_{f}}\left(1+\sin \theta_{0}\right) .
$$

Equation (4) depends on $x_{f}: \Delta \beta_{\mathrm{opt}}$ decreases when increasing the distance between the target and best focus. This allows us to relax the experimental constraint of obtaining large WFR velocity [30]. However, there is a trade-off since at larger values of $x_{f}$ the intensity of the laser at the surface decreases. For the largest value we investigate, $x_{f}=50 \lambda_{0}$ [where Eq. (4) gives $\Delta \beta_{\mathrm{opt}} \simeq 30 \mathrm{mrad}$ ], the laser field amplitude on target is decreased by $8 \%$ with respect to the configuration studied below, $x_{f}=25 \lambda_{0}$ where $\Delta \beta_{\mathrm{opt}} \simeq 60 \mathrm{mrad}$.

An additional improvement on the interaction setup was made by considering that both the efficient excitation and propagation of SPW strongly depend on the grating and surface properties. By an extensive numerical study of the effect of the target profile on the SPW excitation [31], we have found that the best coupling is obtained for a blazed grating, as also suggested experimentally in Ref. [21]. A systematic comparison between targets fully modulated or only partially engraved showed that a partially engraved target (with grooves only in the laser-irradiated spot) efficiently mitigates radiation losses due to scattering of the SPW off the grating. The use of this mixed surface grating allows a better propagation of the SPW along the flat surface. In our simulations (not shown), we observed an increase of $25 \%$ of the maximum electron energy using such targets.

To test our claims, two series of 2D3V PIC simulations were performed with the code SMILEI [32] considering different laser field strengths $a_{0}=e E_{0} /\left(m_{e} c \omega_{0}\right)$. First, a nonrelativistic laser intensity $a_{0}=0.1$ allows us identify the optimal parameters for SPW excitation. Then, the UHI regime of interaction $a_{0}=5$ and electron acceleration along the target surface are considered. In both cases, the general setup of the simulation is given in Fig. 1 with numerical parameters. ${ }^{2}$ The grating target, of thickness $3 \lambda_{0}$, has density $n=100 n_{c}$, ion to electron mass ratio $m_{i} /\left(m_{e}\right)=1836$, and temperature ratio $T_{i} /\left(T_{e}\right)=0.1$ with $T_{e}=50 \mathrm{eV}$. The periodicity of the

\footnotetext{
${ }^{1}$ In PIC simulations, $v_{\mathrm{sl}}$ is measured by locating the position of the maximum laser field amplitude as a function of time at the target surface and time averaging over the laser high frequency.

${ }^{2}$ The simulation box is $39 \lambda_{0} \times 72 \lambda_{0}$ (in the $x-y$ directions), with $9984 \times 18432$ cells (spatial resolution $\Delta=\lambda_{0} / 256$ ), and time resolution $\Delta t=0.95 \Delta / \sqrt{2}$. Electromagnetic field boundary conditions are injecting and absorbing in $x$ and periodic in $y$. Particle boundary conditions in $x$ are reflecting (left) or thermalizing (right), and periodic in $y$. There are 32 macroparticles per species per cell.
} 

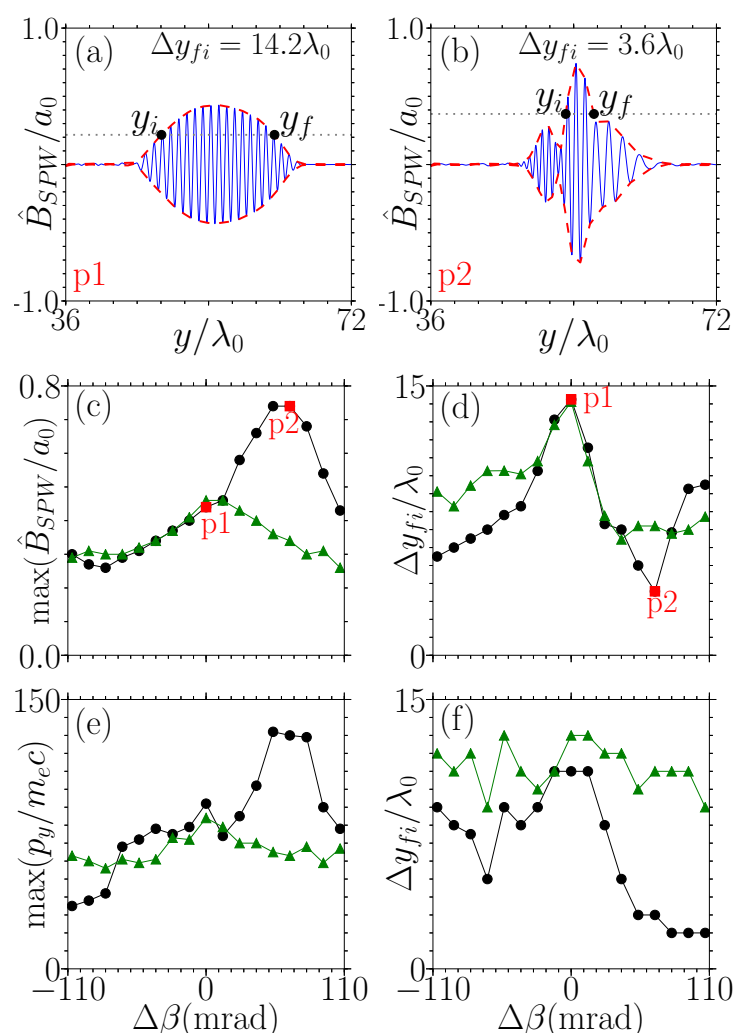

FIG. 2. SPW magnetic field at the target surface for (a) $\Delta \beta=0$ and (b) $\Delta \beta=67 \mathrm{mrad}$ with $a_{0}=0.1$ and $x_{f}=25 \lambda_{0}$. (c) Maximum SPW field amplitude and (d) duration (FWHM) vs the WFR parameter $\Delta \beta$ for $a_{0}=0.1$. Markers $\mathrm{p} 1$ and $\mathrm{p} 2$ indicate the cases shown in panels (a) and (b). (e) Maximum electron momentum along the surface $\left(p_{y}\right)$ and (f) electron bunch duration (FWHM) vs $\Delta \beta$ for $a_{0}=5$. In panels (c) to (f), $x_{f}=0$ (green triangles) and $x_{f}=25 \lambda_{0}$ (black circles).

grating is $d=2 \lambda_{0}$ with groove depth $h=0.44 \lambda_{0}$ and blazed angle $\phi_{b}=13^{\circ}$. A flat surface (at $y>42 \lambda_{0}$ ) follows the grating so that the laser illuminates only the number of ripples corresponding to the projected pulse waist onto the surface. The driving laser is a $p$-polarized Gaussian pulse with transverse size $w_{\perp}=5.2 \lambda_{0}$, duration [full width at half maximum (FWHM) in intensity] $T=10 \lambda_{0} / c{ }^{3}$ The laser pulse impinges onto the grating target at the resonant angle $\theta_{0}=31^{\circ}$. The simulation is run up to time $t_{0}+20 \lambda_{0} / c$, with $t_{0}$ being the time when the peak of the pulse reaches the target. Unless specified otherwise, all values are taken at the end of the simulation.

We first consider $a_{0}=0.1$ for which relativistic nonlinearities can be neglected. The $z$ component of the magnetic field, ${ }^{4}$ noted $B_{\mathrm{SPW}}\left[\right.$ or $\left.\hat{B}_{\mathrm{SPW}}=e B_{\mathrm{SPW}} /\left(m_{e} \omega_{0}\right)\right]$, is taken as representative of the SPW, with all the other field components being proportional to it. For $n \gg n_{c}$, and in the vacuum

\footnotetext{
${ }^{3}$ The laser transverse profile is Gaussian, $F\left(y^{\prime}\right)=\exp \left(-y^{2} / w_{\perp}^{2}\right)$ with $w_{\perp}=5.2 \lambda_{0}$ and its time profile is $\cos ^{2}: f(t)=\cos (\pi t /(2 T))$ for $|t|<T$ (0 otherwise), with $T=10 \lambda_{0} / c$.

${ }^{4} B_{\mathrm{SPW}}$ is collected at $t=t_{0}+20 \lambda_{0} / c$, on flat surface far from the laser-plasma interaction zone. The magnetic field has been filtered, selecting values of $k>2 k_{\mathrm{SPW}}$.
}

side, the linear approximation yields $\left|E_{x}\right| \sim c\left|B_{\mathrm{SPW}}\right|$ and $\left|E_{y}\right| \sim$ $c\left|B_{\text {SPW }}\right| \sqrt{n_{c} / n}$.

In Fig. 2, we show a snapshot of $\hat{B}_{\mathrm{SPW}}$ (blue solid line) and its envelope (red dashed line) along the target surface for $x_{f}=25 \lambda_{0}$, (a) $\Delta \beta=0$, and (b) $\Delta \beta=67 \mathrm{mrad}$. The latter case corresponds to the most intense and shortest SPW found in our simulations, $\Delta \beta_{\text {opt }}=60 \mathrm{mrad}$. With this optimal WFR parameter, the SPW peak amplitude is increased by $\approx 65 \%$ with respect to the case without WFR and its duration, measured as the signal FWHM, is reduced by four from 14.2 to $3.6 \lambda_{0} / c$.

Figures 2(c) and 2(d) show the maximum value of $\hat{B}_{\mathrm{SPW}}$ and the measured SPW duration as the result of a parametric scan of $\Delta \beta$ for $x_{f}=0$ (target at focus, green triangles) and $x_{f}=25 \lambda_{0}$ (target off-focus, black circles). At focus, WFR has a small impact on the SPW excitation: The most intense SPW is obtained for $\Delta \beta=0$, and using nonzero $\Delta \beta$ decreases the duration of the SPW but also its maximum amplitude. Instead, for $x_{f}=25 \lambda_{0}, \Delta \beta$ acts as a tuning parameter, allowing both to shorten the SPW and to increase its amplitude. We observe the shortest and most intense SPW for $\Delta \beta \simeq 67 \mathrm{mrad}$. This is in good agreement with the optimal prediction from Eq. (4), $\Delta \beta_{\text {opt }} \simeq 60 \mathrm{mrad}$. Note a smooth trend around this optimal value; the point directly on the left of $\mathrm{p} 2$ corresponds to $\Delta \beta=53 \mathrm{mrad}$. Interestingly, even though the on-target laser intensity is reduced when increasing $x_{f}$ to $25 \lambda_{0}$, a significant increase of the SPW amplitude is still obtained using the optimal WFR parameter. A parametric scan considering $x_{f}=$ $50 \lambda_{0}$ (not shown) leads to an optimal WFR parameter $\Delta \beta \simeq$ $33 \mathrm{mrad}$ also in good agreement with $\Delta \beta_{\mathrm{opt}}=30 \mathrm{mrad}$ from Eq. (4). Finally, as expected positive values of $\Delta \beta$, for which the sliding velocity is along the SPW propagation direction, give a maximal effect. In contrast, for negative $\Delta \beta$, the SPW is still of a shorter duration but with a reduced amplitude, roughly that obtained when placing the target at best focus.

We now turn our attention to the second series of simulations performed in the UHI regime $\left(a_{0}=5\right)$ and electron acceleration. The bottom row in Fig. 2 shows [Fig. 2(e)] the maximum electron momentum parallel to the surface and [Fig. 2(f)] the characteristic width ${ }^{5}$ of the accelerated electron bunch as a function of $\Delta \beta$, considering $x_{f}=0$ (green triangles) and $x_{f}=25 \lambda_{0}$ (black circles). Both panels exhibit very similar features to those observed at low intensity. Placing the target at focus $\left(x_{f}=0\right)$, the accelerated electron bunch maximum energy and duration are marginally affected by WFR. In contrast, for $x_{f}=25 \lambda_{0}$, WFR significantly impacts electron acceleration: Taking $\Delta \beta>0$ leads to more energetic, shorter electron bunches. By comparing the case for which the target is at focus with $\Delta \beta=0$ and that with the target at $x_{f}=25 \lambda_{0}$ with $\Delta \beta=67 \mathrm{mrad}$, one finds an increase of the maximum electron momentum by $62 \%$ [from $\max \left(p_{y}\right) \simeq$ $80 m_{e} c$ to $\left.\simeq 130 m_{e} c\right]$ and much shorter bunches when the optimal (positive) WFR parameter is considered and target is off focus. The optimum value $\Delta \beta=67 \mathrm{mrad}$ found for electron acceleration in this regime is the same as found earlier for efficient, ultrashort SPW excitation at lower intensity.

\footnotetext{
${ }^{5}$ The duration of the electron bunch is estimated from its spatial width through the relation $\Delta \tau_{f i} \simeq \Delta y_{f i} / c$.
} 


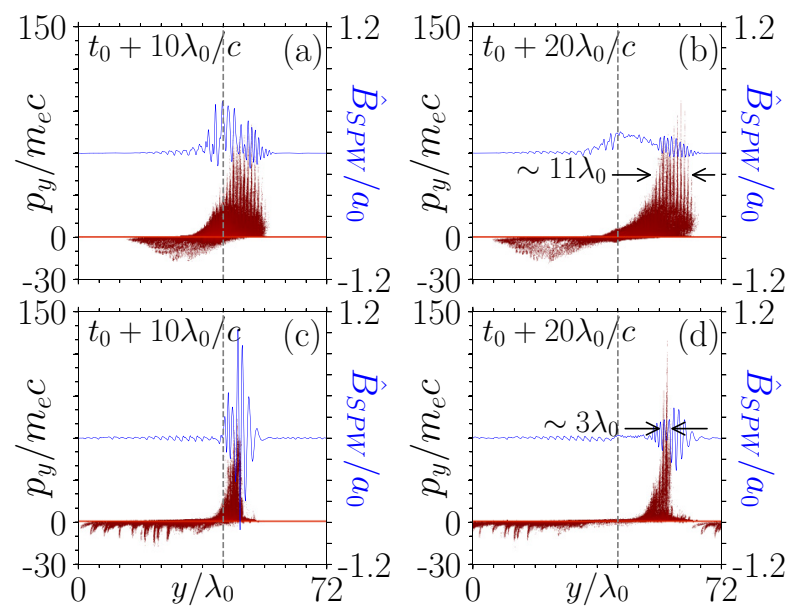

FIG. 3. Electron phase-space (red dots) and SPW field amplitude (blue line, right scale) for $a_{0}=5$ at times, $t=t_{0}+10 \lambda_{0} / c$ and $t=t_{0}+20 \lambda_{0} / c$. [(a), (b)] $\Delta \beta=0$ and [(c), (d)] $\Delta \beta=67 \mathrm{mrad}$. The gray vertical dashed line indicates the end of the grating and beginning of the flat region.

Figure 3 gives further insights into the acceleration process. The electron phase space and SPW magnetic field at the target surface $\left(x_{f}=25 \lambda_{0}\right)$ are shown at two different times, for [Figs. 3(a) and 3(b)] $\Delta \beta=0$ and [Figs. 3(c) and 3(d)] $\Delta \beta=67 \mathrm{mrad}$ (optimal condition). In both cases, the duration of the electron bunch is proportional to the duration of the SPW, the shortest SPW obtained for $\Delta \beta=$ $67 \mathrm{mrad}$ leading to the shortest electron bunch. For $\Delta \beta=0$ [Figs. 3(a) and 3(b)], the SPW is strongly damped at $t=$ $t_{0}+20 \lambda_{0} / c$ : The electron bunch has reached its parallel momentum $\max \left(p_{y}\right) \simeq 90 m_{e} c$ and has a width (measured from the FWHM in momentum) of $\Delta y_{f i}=11 \lambda_{0}$. The acceleration process is more efficient using the optimal WFR parameter $\Delta \beta=67 \mathrm{mrad}\left[\right.$ Figs. 3(c) and 3(d)]. At $t=t_{0}+10 \lambda_{0} / c$, two periods after the laser has left the surface, the magnetic field is intense $\left(\hat{B}_{\mathrm{SPW}} \simeq 1.2 a_{0}\right)$ and the most energetic electrons have already reached momentum up to $\max \left(p_{y}\right) \simeq 70 m_{e} c$. Ten periods later, a narrow $\left(\Delta y_{f i}=3 \lambda_{0}\right)$ and energetic $\left[\max \left(p_{y}\right) \simeq\right.$ $130 m_{e} c$ ] electron bunch is obtained, while the SPW has been significantly damped.

Similar observations can be drawn from Fig. 4. In Fig. 4(a), the electron distribution in energy and direction (the angle is defined in the simulation plane with respect to the $x$ axis) is shown, demonstrating that the most energetic electrons are accelerated mainly along the target's surface and in the $y>0$ direction (i.e., in the SPW direction of propagation). Figure 4(b) shows the energy distribution of the electron, for different values of $\Delta \beta$.

These results and in particular the increase of the maximum electron energy (equiv. momentum) are consistent with what one expects from the increase of the SPW amplitude by use of the WFR driving pulse. Indeed, an upper limit of the electron energy gain in the SPW has been derived in Ref. [18] by generalizing the results of wake-field acceleration [33,34], leading $\Delta \mathcal{E} \sim \chi \gamma_{\phi} \max \left|\hat{B}_{\mathrm{SPW}}\right| m_{e} c^{2}$ so that $\Delta \mathcal{E}$ is proportional to the SPW field amplitude. Here $\gamma_{\phi}=\left(1-v_{\phi}^{2} / c^{2}\right)^{-1 / 2}$ and $\chi$ is a constant of order one, reaching at most 4 [18]. In our simulations, the magnetic field of the SPW (time-averaged
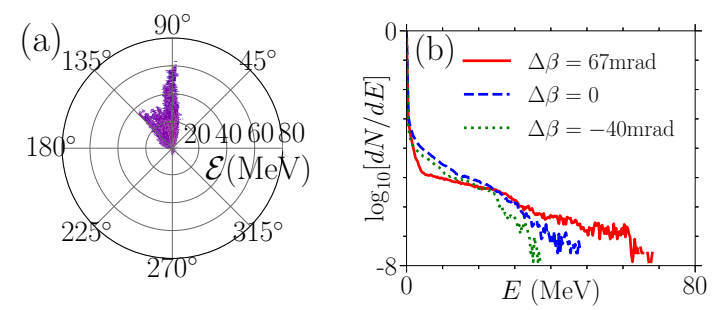

FIG. 4. (a) Electron energy distribution in $\mathrm{MeV}$ as a function of the emission angle $\phi=\tan ^{-1}\left(p_{y} / p_{x}\right)$ for $\Delta \beta=67 \mathrm{mrad}$ and $a_{0}=5$. (b) Electron energy distribution for $\Delta \beta=67$ (red solid line), $\Delta \beta=0$ (blue dashed line), and $\Delta \beta=-40 \mathrm{mrad}$ (green dotted line).

over the wave period) reached at most $\max \left|\hat{B}_{\mathrm{SPW}}\right| \approx 3.8$ for $\Delta \beta=0$ and $\max \left|\hat{B}_{\mathrm{SPW}}\right| \approx 7.0$ for $\Delta \beta=67 \mathrm{mrad}$. Considering that $\gamma_{\phi} \simeq 10$ for $n=100 n_{c}$, we then obtain the upper limit $\Delta \mathcal{E} \simeq 154 m_{e} c^{2}$ for $\Delta \beta=0$ and $\Delta \beta=67 \mathrm{mrad}$ for $\Delta \mathcal{E} \simeq$ $280 m_{e} c^{2}$. These predictions overestimate the electron energy as they assume (i) no wave decay over the distance required for acceleration, (ii) optimal electron injection, and (iii) acceleration exactly parallel to the target surface, while it has been observed that electrons are deflected in the perpendicular direction [19].

To gain further insight into the acceleration process, we performed a particle tracking of the most energetic electrons and evaluated the trajectory-averaged value of the longitudinal field $\left\langle E_{y}\right\rangle$ acting on the particle. This allows to define an acceleration length $l_{\mathrm{acc}}=\Delta \mathcal{E} /\left|e\left\langle E_{y}\right\rangle\right|$. From the particle track, we found $\Delta \mathcal{E} \simeq 90 m_{e} c^{2}$ and $\left\langle E_{y}\right\rangle \simeq-1.0 m_{e} c \omega_{0} / e$ for $\Delta \beta=$ 0 , and $\Delta \mathcal{E} \simeq 130 m_{e} c^{2}$ and $\left\langle E_{y}\right\rangle \simeq-1.4 m_{e} c \omega_{0} / e$ for $\Delta \beta=$ $67 \mathrm{mrad}$. In both cases, this leads to an acceleration length $l_{a c c} \sim 15 \lambda_{0}$, consistent with the observed particle trajectories. This length largely exceeds the laser spot size and is close to the length over which the SPW decreases its amplitude significantly (see, e.g., Fig. 3). This confirms the electrons are accelerated by the SPW as it propagates along the target surface.

In the optimal case, the highest energy particles (in the range 30-70 MeV) form a bunch with duration of $\simeq 3 \lambda_{0} / c$ $\left[\sim 8\right.$ fs for $\left.\lambda_{0}=0.8 \mu \mathrm{m}\right]$ and total charge $\simeq 10 \mathrm{pC} / \lambda_{0}$ (in our $2 \mathrm{D}$ simulations). Assuming a bunch width (in the $z$ direction) of the order of the laser pulse with $w_{\perp}=5.2 \lambda_{0}$, one could expect few cycles of electron bunches with a charge of $\approx 52 \mathrm{pC}$. These results are competitive with cutting-edge laser wake-field electron beams from underdense plasmas. Considering similar laser parameters and electron energies, short, high-charge electron bunches were obtained with energy $85 \mathrm{MeV}$ (21 MeV energy spread), total charge $15 \mathrm{pC}$, and duration $4.4 \mathrm{fs}$ [35].

In conclusion, a laser with WFR and an appropriately tailored plasma target allow us to control the duration and amplitude of SPW in the linear and relativistic regime. As a consequence, ultrashort (near single cycle), energetic and highly charged electron bunches are generated. The optimal parameters are clearly identified; since they are well within the capabilities of current UHI installations, this work opens new prospects and provide guidelines for forthcoming experiments. 
Financial support from Grant No. ANR-11-IDEX-000402 Plas@Par is acknowledged. Simulations were performed on the Irene-SKL machine hosted at TGCC- France, using High Performance Computing resources from GENCITGCC (Grant No. 2018-x2016057678) and PRACE (Project
MIMOSAS). P.S.K. was supported by the CEA NUMERICS program, which has received funding from the European Union's Horizon 2020 research and innovation program under the Marie Sklodowska-Curie Grant agreement No. 800945.
[1] H. Raether, Surface Plasmons on Smooth and Rough Surfaces and Gratings (Springer-Verlag, Berlin, 1988).

[2] W. Barnes, A. Dereux, and T. Ebbesen, Nature (London) 424, 824 (2003).

[3] S. A. Maier, Plasmonics: Fundamentals and Applications (Springer-Verlag, Berlin, 2007).

[4] J. M. Pitarke, V. M. Silkin, E. V. Chulkov, and P. M. Echenique, Rep. Prog. Phys. 70, 1 (2007).

[5] P. K. Jain, X. Huang, I. H. El-Sayed, and M. A. El-Sayed, Acc. Chem. Res. 41, 1578 (2008).

[6] K. C. Y. Huang, M.-K. Seo, T. Sarmiento, Y. Huo, J. S. Harris, and M. L. Brongersma, Nat. Photon. 8, 244 (2014).

[7] E. Ozbay, Science 311, 189 (2006).

[8] T. Chung, S.-Y. Lee, E. Y. Song, H. Chun, and B. Lee, Sensors 11, 10907 (2011).

[9] G. S. Agarwal and S. S. Jha, Solid State Commun. 41, 499 (1982).

[10] J. L. Coutaz, M. Neviere, E. Pic, and R. Reinisch, Phys. Rev. B 32, 2227 (1985).

[11] P. Jatav and J. Parashar, Phys. Plasmas 26, 022301 (2019).

[12] J. Kupersztych, P. Monchicourt, and M. Raynaud, Phys. Rev. Lett. 86, 5180 (2001).

[13] J. Zawadzka, D. A. Jaroszynski, J. J. Carey, and K. Wynne, Appl. Phys. Lett. 79, 2130 (2001).

[14] M. Raynaud, J. Kupersztych, C. Riconda, J. C. Adam, and A. Héron, Phys. Plasmas 14, 092702 (2007).

[15] T. Ceccotti, V. Floquet, A. Sgattoni, A. Bigongiari, O. Klimo, M. Raynaud, C. Riconda, A. Heron, F. Baffigi, L. Labate et al., Phys. Rev. Lett. 111, 185001 (2013).

[16] A. Bigongiari, M. Raynaud, C. Riconda, and A. Héron, Phys. Plasmas 20, 052701 (2013).

[17] Y. Tian, J. Liu, W. Wang, C. Wang, A. Deng, C. Xia, W. Li, L. Cao, H. Lu, H. Zhang et al., Phys. Rev. Lett. 109, 115002 (2012).

[18] C. Riconda, M. Raynaud, T. Vialis, and M. Grech, Phys. Plasmas 22, 073103 (2015).
[19] L. Fedeli, A. Sgattoni, G. Cantono, D. Garzella, F. Réau, I. Prencipe, M. Passoni, M. Raynaud, M. Květoň, J. Proska et al., Phys. Rev. Lett. 116, 015001 (2016).

[20] L. Fedeli, A. Sgattoni, G. Cantono, and A. Macchi, Appl. Phys. Lett. 110, 051103 (2017).

[21] G. Cantono, A. Sgattoni, L. Fedeli, D. Garzella, F. Réau, C. Riconda, A. Macchi, and T. Ceccotti, Phys. Plasmas 25, 031907 (2018).

[22] A. Macchi, Phys. Plasmas 25, 031906 (2018).

[23] M. Raynaud, A. Héron, and J.-C. Adam, Plasma Phys. Controlled Fusion 60, 014021 (2018).

[24] X. M. Zhu, R. Prasad, M. Swantusch, B. Aurand, A. A. Andreev, O. Willi, and M. Cerchez, High Power Laser Sci. Eng. 8, 15 (2020)

[25] M. Raynaud, A. Héron, and J.-C. Adam, Sci. Rep. 10, 13450 (2020).

[26] G. Cantono, L. Fedeli, A. Sgattoni, A. Denoeud, L. Chopineau, F. Réau, T. Ceccotti, and A. Macchi, Phys. Rev. Lett. 120, 264803 (2018).

[27] F. Pisani, L. Fedeli, and A. Macchi, ACS Photon. 5, 1068 (2018).

[28] P. K. Kaw and J. B. McBride, Phys. Fluids 13, 1784 (1970).

[29] H. Vincenti and F. Quéré, Phys. Rev. Lett. 108, 113904 (2012).

[30] F. Quéré, H. Vincenti, A. Borot, S. Monchocé, T. J. Hammond, K. T. Kim, J. A. Wheeler, C. Zhang, T. Ruchon, T. Auguste et al., J. Phys. B: At. Mol. Opt. Phys. 47, 124004 (2014).

[31] P. S. Kleij, M.Sc. thesis, Università di Pisa, Sorbonne Université, France, 2019, https://etd.adm.unipi.it/t/etd-09022019103844/.

[32] J. Derouillat, A. Beck, F. Pérez, T. Vinci, M. Chiaramello, A. Grassi, M. Flé, G. Bouchard, I. Plotnikov, N. Aunai et al., Comput. Phys. Commun. 222, 351 (2018).

[33] T. Tajima and J. M. Dawson, Phys. Rev. Lett. 43, 267 (1979).

[34] P. Mora and F. Amiranoff, J. Appl. Phys. 66, 3476 (1989); P. Mora, Phys. Fluids 4, 1630 (1992).

[35] O. Lundh, J. Lim, C. Rechatin, L. Ammoura, A. Ben-Ismail, X. Davoine, G. Gallot, J.-P. Goddet, E. Lefebvre, V. Malka, J. Faure et al., Nat. Phys. 7, 219 (2011). 